\title{
Nanostructured biomaterials
}

Jacques Livage

Laboratoire Chimie de la Matière Condensée, Collège de France, 11 place Marcelin Berthelot, 75231 Paris Cedex 05, France Collège de France,

Inorganic crystals are precipitated from solutions via a nucleation and growth process. They exhibit regular shapes that mainly depend on their crystal symmetry. Biomineralization usually lead to more sophisticated shapes. Bio-molecules are involved in the formation of the inorganic phase. They prevent the growth of large crystals and favor the self assembly of nanocrystals. Nanostructured biomaterials are then formed that offer specific physical properties. The formation of calcium carbonate shells by coccolithes will be taken as an example. These solid shells are made in order to protect these single cell micro-algaes against predators. They are formed via the self-assembly of $\mathrm{CaCO}_{3}$ nanocrystals. These nanostructured shells then exhibit specific optical properties that control the photosynthetic activity of entrapped cells. 